\title{
Propuesta de Una Escala de Valoración de Las Funciones Ejecutivas en Universitarios
}

\author{
Proposal For An Evaluation Scale Of Executive Functions \\ In University Students
}

María Judith López-Cárdenas, Carlos Ramos-Galarza, ${ }^{1,2}$

\section{Resumen}

Introducción. Las funciones ejecutivas constituyen un conjunto de procesos cognitivos, afectivos y motivacionales que permiten el control consciente del pensamiento, el desarrollo de tareas de gran complejidad y la facilitación a la adaptación de nuevas situaciones como es característico en el contexto universitario. Objetivo. Proponer una escala que valore las funciones ejecutivas en el contexto de educación superior mediante la ejecución de un estudio psicométrico con estudiantes entre 18 y 25 años de edad. Sujetos y método. Participaron 752 estudiantes universitarios de Quito-Ecuador en edades comprendidas entre los 18 y 25 años de edad, de los cuales 498 (66.2\%) son de género femenino y 254 (33.8\%) de género masculino. Como reactivo se propuso una escala para valorar las funciones ejecutivas en auto reporte. Resultados. El análisis factorial exploratorio reportó una organización de 8 funciones ejecutivas que explican el $57 \%$ de la varianza del constructo $(\mathrm{p}=<.001)$. Se obtuvo un adecuado nivel de fiabilidad entre $\alpha=.70$ y .86, se eliminó un factor puesto que su nivel de confiabilidad fue muy bajo y no mejoraba ante ningún procedimiento estadístico. La correlación entre los factores propuestas fue adecuada en una magnitud mediana $\mathrm{r}=.67$ y .39. Conclusión. Los resultados exponen que la escala propuesta está basada en situaciones de la vida cotidiana del estudiante, este diseño de escala permite identificar situaciones tanto patológicas como condiciones que afecten la calidad de vida y desenvolvimiento en el ámbito universitario. Además, los indicadores psicométricos aportan evidencia en favor de su uso fiable en el contexto universitario.

Palabras clave: Funciones Ejecutivas, Escala de Auto reporte, Estudiantes Universitarios

\section{Abstract}

Introduction. The executive functions constitute a set of cognitive, affective and motivational processes, which allow the conscious control of thought, development of highly complex tasks, that is going to facilitate the adaptation to new situations, it is therefore necessary the contribution of new evaluation methodologies of executive functions centered on the university population, this is going to facilitate the access to check the cognitive processes of the pre-frontal cortex. Objective. Evaluate executive functions in university students between 18 and 25 years of age using a self-report scale. Subjects and method. 752 university students from Quito-Ecuador participated, aged between 18 and 25 years old, of whom 498 (66.2\%) were female and 254 (33.8\%) were male. As measuring reagent, the scale used to evaluate the executive functions in self-report was proposed in the project: Neuropsychological Systems for Supervision of Cognition and Behavior for the Benefit of Managing the Learning of Higher Education Students. Results. Exploratory factor analysis reported an organization of 8 executive functions that explain 57\% of the variance of the construct $(\mathrm{p}=<.001)$. An adequate level of reliability was obtained between $\alpha=.70$ and .86 , a factor was eliminated since its level of reliability was very low and did not improve under any statistical procedure. The correlation between the proposed factors was adequate at a median magnitude $r=.67$ and .39 . Conclusion. The results show that the proposed scale is based on situations in the student's daily life, this scale design allows identifying both pathological situations and conditions that affect the quality of life and development in the university environment. Furthermore, psychometric indicators provide evidence in favor of their reliable use in the university context.

Keywords: Executive Functions, Self-report Scale, University Students

Rev. Ecuat. Neurol. Vol. 29, N³, 2020

'Facultad de Psicología, Pontificia Universidad Católica del Ecuador. Quito, Ecuador

${ }^{2}$ Centro de Investigación en Mecatrónica y Sistemas Interactivos MIST y Carrera de Psicología, Universidad Tecnológica Indoamérica de Ecuador. Quito, Ecuador
Correspondencia:

Carlos Ramos-Galarza, Ph.D. Facultad de Psicología, Pontificia Universidad Católica del Ecuador, Av. 12 de Octubre 1076 y Roca, Quito, Ecuador. https://orcid.org/0000-0001-5614-1994

E-mail: caramos@puce.edu.ec 


\section{Introducción}

Las funciones ejecutivas (FE) son denominadas, en el campo de la neuropsicología, como procesos en los que se asocian ideas, movimientos y acciones que se encuentran dirigidos tanto al desarrollo de las actividades del ser humano, como a la resolución de problemas! La autoría del término de FE se relaciona con el trabajo realizado por Muriel Lezak, quien propuso que estas funciones son capacidades que tiene el ser humano para formular objetivos, planear y realizar proyectos de modo efectivo? Por otro lado, se ha mencionado que el estudio de las funciones ejecutivas fue descrito originalmente por Harlow, en el año 1868, en donde argumentó mediante un análisis excepcional de su paciente Phineas Gage, la presencia de cambios cognoscitivos, variaciones en la conducta y la personalidad, el mismo había sufrido un accidente que le provocó una lesión en los lóbulos frontales.

Otro autor que inició con el estudio de las habilidades del lóbulo frontal fue Alexander Luria quien vinculó a los lóbulos frontales la habilidad de inhibición de las respuestas inmediatas, la programación motora, la orientación de las conductas acorde a las consecuencias de las acciones realizadas, la integración de la conducta, la personalidad y la conciencia! Además, gracias a las aproximaciones neuropsicológicas, a los estudios en neuro-imagen funcional y al reporte de personas con lesiones en el área prefrontal, se ha logrado comprender el papel fundamental de las funciones ejecutivas en los lóbulos frontales como integradoras de procesos de planificación, organización y regulación del comportamiento y la cognición, que permiten llevar a cabo actividades voluntarias y complejas?

Las funciones ejecutivas se sitúan en la cima del desarrollo cerebral, en el nivel jerárquico más elevado de actividad mental ya que actúan como un aparato integrador capaz de articular todas las piezas que regulan la actividad mental superior, las mismas incluyen mecanismos de unificación que permiten enlazar la información del pasado y el presente, otorgando opciones de respuesta en el futuro.

Entre los modelos teóricos de las funciones ejecutivas se encuentran diversas propuestas, como el sistema de supervisión atencional (SSA) propuesto por Sallice y Burgess, en el cual destaca la relevancia del sistema prefrontal para el procesamiento de la información, facilitando el apropiado control atencional frente a tareas nuevas $;{ }^{4}$ el modelo de marcador somático, propuesto por Damasio ${ }^{5}$ el cual explica de qué manera influye las emociones en la toma de decisiones y el razonamiento; Stuss y Benson, propusieron un modelo explicativo jerárquico de las funciones ejecutivas, en donde se ven integradas las funciones de alto nivel como funciones independientes pero interconectadas entre sí.
En el modelo de memoria de trabajo y funciones ejecutivas, ${ }^{4}$ se menciona que la memoria de trabajo vendría a ser el brazo activo de las funciones ejecutivas, ya que es un elemento esencial del sistema atencional operativo, el cual permite trabajar con contenidos de la memoria guiando al comportamiento a un fin u objetivo. En el modelo de las funciones FE e inteligencia, gracias a la teorización de Gardner ${ }^{6}$ sobre las inteligencias múltiples y Goldberg $^{7}$ con la propuesta de la inteligencia ejecutiva o "factor i," se genera el reconocimiento de patrones esenciales para el desarrollo y funcionamiento adecuado del área prefrontal.

Por otro lado, los modelos factoriales propuestos por Miyake y colaboradores, analizan y exponen que existen seis factores que forman el conglomerado básico de las funciones ejecutivas: actualización, flexibilidad, inhibición, planificación, toma de decisiones y fluencia o velocidad de procesamiento. De igual modo se encuentra la Teoría del Filtro Dinámico, en donde Shimamura, ${ }^{9}$ menciona que existen cuatro aspectos del control ejecutivo que se encargan del proceso de filtrado para controlar y monitorizar la información, estas serían: la selección, mantenimiento, actualización y redirección. Además, Koechiln, plantea el Modelo de los Ejes Diferenciales en el Control Ejecutivo, el cual refiere que las funciones cognitivas menos complejas dependen de zonas posteriores y conforme van aumentando el nivel de complejidad dependen de áreas anteriores. ${ }^{10}$

Gracias a la teorización que gira en torno a las funciones ejecutivas, se puede recalcar la importancia de estas en las actividades que desempeñan los estudiantes y la necesidad de su evaluación. A lo largo de las diversas carreras universitarias, se logra observar que ciertos estudiantes presentan dificultades en torno a la organización y planificación de sus actividades, al control de sus emociones, a la flexibilidad o adaptación a tareas nuevas, a la capacidad atencional y toma de decisiones o la habilidad de culminar de manera independiente sus tareas universitarias." ${ }^{11}$

Según Ramos-Galarza y colaboradores ${ }^{11}$ existe una relación entre las funciones ejecutivas y el rendimiento académico en universitarios, los mismos demostraron que existe una asociación de mediana magnitud entre el rendimiento académico y las dificultades en la supervisión consciente del comportamiento y la memoria de trabajo. Y una relación de magnitud media a grande entre todos los componentes de las FE y el comportamiento de los alumnos. Además, demostraron que las funciones ejecutivas explican $31 \%$ de la varianza de rendimiento académico. Por otro lado, Villegas ${ }^{12}$ demostró que existe una relación entre funciones ejecutivas de control emocional, organización de materiales, iniciativa y memoria de trabajo con la aparición de estrés académico, esto sugiere la necesidad evaluativa para reconocer las dificultades que puedan estar presentando los estudiantes. 
Estas habilidades son parte del control ejecutivo del lóbulo frontal que se desarrollará desde etapas tempranas del individuo, que permitirán, en un futuro, presentar un óptimo desempeño académico. Entre los hitos del proceso madurativo de las FE, se pueden destacar los siguientes: se conoce que, entre los 20 y 25 años de vida, se consolida como elemento rector de los procesos cognitivos, las funciones ejecutivas. ${ }^{4} \mathrm{Y}$ entre los 18 y 29 años son capaces de solucionar problemas, planear actividades y una óptima generación verbal. Se puede decir entonces que las características de un adulto joven y adulto medio deberían ser, una organización cognoscitiva esperable y funcional. Dentro de esta investigación, al abordar estudiantes entre los 18 y 25 años de edad, se puede decir que se encuentran en la culminación del desarrollo y maduración de sus funciones ejecutivas, en donde se esperaría que un porcentaje logre autorregular su conducta y pueda establecer, supervisar, mantener, corregir y alcanzar un objetivo.

Se ha considerado que el estudio de las FE en estudiantes universitarios debería tener una mayor incidencia en el ámbito investigativo, ya que es de gran importancia que este sea valorado mediante una escala que muestre el desempeño de las mismas, debido a que no solo se apunta hacia el nivel de desarrollo de aprendizajes académicos, sino también incluye el desarrollo de competencias sociales que ayudan a los universitarios a encontrar formas óptimas de adaptación hacia las exigencias de la sociedad. La investigación, además, ayudaría a reconocer los déficits de los alumnos y resolver asuntos de deserción estudiantil o bajo rendimiento académico que podrían devenir del desempeño ejecutivo.

Según Nunes y colaboradores ${ }^{13}$ la evaluación de las funciones ejecutivas es un aspecto todavía en construcción en esta línea de investigación, debido a la falta de consenso metodológico con respecto a los procesos evaluativos. Las autoras realizaron un análisis de los instrumentos más utilizados para evaluar las funciones ejecutivas en los últimos 5 años, en donde a pesar de encontrar publicaciones de investigaciones comparativas, correlaciónales y de validación, comprueban y afirman que existe una falta de instrumentos que evalúen los dominios de las funciones ejecutivas y la necesidad de construir nuevos reactivos con esta finalidad.

En el estudio de las funciones ejecutivas todavía no se logra una claridad absoluta sobre sus procesos evaluativos y existe una carencia evidente de instrumentos que las evalúen en el ámbito universitario. En general, se presupone que los test neuropsicológicos utilizados para la evaluación de las funciones ejecutivas valoran dichos procesos cognitivos, empero, el origen de los procesos ejecutivos implicados en el desempeño de estas tareas no está clara, del mismo modo, los términos para consolidar y clasificar estas habilidades mentales, tiene una pobre validez de constructo, ya que el origen del marco teórico puede ser variado desde las posturas de diversos autores ${ }^{14}$ Del mismo modo, Portellano y García ${ }^{4}$ mencionan que hay una gran demanda por evaluar funciones ejecutivas en diversas aristas, no obstante, actualmente existe una carencia de escalas o test que estén diseñadas o adaptadas a la población objeto.

Es por esto que con esta investigación se aporta para solucionar esta carencia, ya que se proporciona una escala fiable que evalúe la cotidianidad de los universitarios en donde se puede ver en acción las funciones ejecutivas. En este instrumento se encuentran ítems claros y precisos al contexto o situación que cualquier universitario podría encontrarse vivenciando, de esta manera acercará al evaluador a una realidad más precisa de lo que sucede con los estudiantes y a partir de allí, proponer métodos de rehabilitación o mejoramiento del control ejecutivo que beneficie a la comunidad universitaria.

\section{Hipótesis de Investigación}

La escala propuesta para la evaluación de las funciones ejecutivas en estudiantes universitarios presentará adecuadas propiedades psicométricas.

\section{Método \\ Participantes}

La muestra consta de 752 estudiantes universitarios de Quito-Ecuador. Según el género 498 (66.2\%) son de género femenino y $254(33.8 \%)$ son de género masculino. En cuanto a la edad, el rango comprendido entre 18 y 25 años. En cuanto al estado civil se encontró que 721 participantes (95.9\%) son solteros, 16 (2.1) casados, $1(0.1 \%)$ divorciado y $4(0.5 \%)$ personas en unión libre. El $95.7 \%$ de los participantes, es decir, 720 personas no tienen hijos, empero el $3.6 \%$ de los participantes restantes si los tiene, 19 (2.5\%) tiene 1 hijo, $2(0.3 \%)$ tienen 2 hijos y $1(0.1 \%)$ participante tiene 3 hijos. En lo que respecta al colegio de procedencia, 549 (73.0\%) participantes provienen de un colegio particular, 152 (20.2\%) de un colegio estatal-fiscal, $49(6.5 \%)$ de un colegio municipal y $2(0.3 \%)$ de un colegio fiscomisional. En cuanto al bachillerato, se encontró que obtuvieron títulos de bachilleres en 16 ramas diferentes.

De los 752 participantes, se evidenció que 57 (7.6\%) viven solos, $671(89.2 \%)$ viven con familiares, 8 (1.1\%) viven con amigos y $12(1.6 \%)$ con pareja. Los evaluados provienen de diferentes universidades del sistema educativo de Ecuador. Las universidades mencionadas se las categoriza por tipos, que son, particular y estatal-fiscal, de los estudiantes 739 (98.3\%) se encontraba en una universidad particular, mientras que $11(1.5 \%)$ se encontraban en una estatal-fiscal. Los estudiantes se encuentran en variadas facultades, carreras y semestres. Por otro lado, $178(23.7 \%)$ de los estudiantes poseen una beca y 553 (73.5) no la tienen. 


\section{Instrumento}

Se realizó una propuesta de escala para valorar las funciones ejecutivas en el contexto educativo universitario, la cual se basa en ítems que analizan estas funciones basadas en situaciones de la vida diaria del contexto universitario. En el anexo 1 se puede observar los ítems de la escala propuesta.

\section{Procedimiento}

La investigación inició luego de la aprobación por parte de un Comité de Ética para la investigación con seres humanos. Los coautores de la investigación se reunieron constantemente para preparar los ítems de la escala, mediante un trabajo colaborativo. Posteriormente se contó con la validación de dos jueces expertos en funciones ejecutivas. Una vez que se tuvo la primera versión de la escala, se ejecutaron cinco entrevistas cognitivas que permitieron identificar aspectos a mejorar en los ítems planteados. Luego, se llevó a cabo un estudio piloto en una muestra de 10 participantes que permitió perfeccionar el contenido de la escala.

Una vez que se tuvo la versión final, se gestionaron autorizaciones en las universidades de Ecuador para realizar las aplicaciones masivas, solicitando colaboración de autoridades y profesores explicando los objetivos y fines de la investigación. Posteriormente, se invitó a los estudiantes a participar voluntariamente, mediante la firma del consentimiento informado, documento que explicaba los objetivos del estudio, el tiempo de duración, indicaciones para responder el instrumento y el resguardo de la confidencialidad, tanto de los datos como de los resultados obtenidos. Dicha aplicación se realizó de forma colectiva en las mismas salas de clases.

\section{Plan de análisis de datos}

El procedimiento fue realizado con estadística descriptiva y se realizó un análisis inicial de las variables sociodemográficas. Se aplicó un análisis factorial exploratorio para analizar la organización de los ítems de la escala. Se aplicó el coeficiente de alfa de cronbach para visualizar la confiabilidad de cada una de las funciones ejecutivas construidas por los ítems. Se aplicó la correlación de Pearson para ver la relación entre las funciones ejecutivas y sus ítems. Todos los análisis se realizaron en el programa SPSS versión 25.

\section{Resultados}

A continuación, se presentará el análisis de los resultados en el siguiente orden: primero el análisis descriptivo de los datos; segundo, el análisis de consistencia interna de cada escala propuesta; tercero, el análisis de correlación entre ítems y, por último, el análisis factorial exploratorio, que presenta 8 factores que explican el 57\% de la varianza de la organización de las funciones ejecutivas en la escala propuesta.

\section{Análisis descriptivo de los datos}

En esta sección se podrá evidenciar la descripción estadística de los datos obtenidos en la investigación. En la tabla 1 se indican los valores estadísticos descriptivos de cada uno de los sistemas propuestos.

Tabla 1. Análisis descriptivo de datos.

\begin{tabular}{lccccc|}
\hline & N & Mínimo & Máximo & Media & DE \\
\hline $\begin{array}{l}\text { Sistema ejecutivo de } \\
\text { control atencional para } \\
\text { tareas }\end{array}$ & 752 & 9.00 & 40.00 & 29.63 & 5.69 \\
\hline $\begin{array}{l}\text { Sistema de supervisión y } \\
\text { monitorización de la con- } \\
\text { ducta para el aprendizaje }\end{array}$ & 752 & 9.00 & 35.00 & 29.77 & 4.47 \\
\hline $\begin{array}{l}\text { Sistema regulador } \\
\text { consciente y voluntario } \\
\text { de las emociones }\end{array}$ & 752 & 8.00 & 35.00 & 25.34 & 4.86 \\
\hline $\begin{array}{l}\text { Sistema de verificación } \\
\text { de la conducta para el } \\
\text { aprendizaje }\end{array}$ & 752 & 4.00 & 20.00 & 16.24 & 3.01 \\
\hline $\begin{array}{l}\text { Sistema de regulación } \\
\text { consciente de la conducta }\end{array}$ & 752 & 4.00 & 20.00 & 16.40 & 2.76 \\
\hline $\begin{array}{l}\text { Sistema de organización } \\
\text { de elementos para } \\
\text { resolver tareas }\end{array}$ & 752 & 4.00 & 20.00 & 15.89 & 3.16 \\
\hline $\begin{array}{l}\text { Sistema ejecutivo para } \\
\text { toma de decisiones }\end{array}$ & 752 & 4.00 & 20.00 & 15.78 & 2.71 \\
\hline $\begin{array}{l}\text { Sistema de control } \\
\text { consciente de impulsos } \\
\text { automáticos }\end{array}$ & 752 & 3.00 & 15.00 & 11.15 & 2.15 \\
\hline
\end{tabular}

$\mathrm{DE}=$ Desviación estándar

\section{Análisis de consistencia interna}

Seguidamente se podrá observar las estadísticas de fiabilidad de cada sistema propuesto, es decir, se analizará la media, la varianza, la correlación y el alfa de Cronbach de cada subescala. Es importante recordar que el Alfa de Cronbach oscila entre 0 y 1 , cuando más cercano esté a 1, mayor consistentes serán los ítems entre sí. El valor mínimo admisible para el coeficiente alfa de Cronbach es .70, por debajo de ese valor la consistencia interna de la escala utilizada es baja15, 16.

La primera escala, denominada Sistema ejecutivo de control atencional para tareas contiene 8 elementos en los cuales se ha obtenido un Alfa de Cronbach de .86 (ver tabla 2).

Tabla 2. Escala 1 Sistema ejecutivo de control atencional para tareas.

\begin{tabular}{|ccccc|} 
& Me (-) & R (-) & C.T & $\boldsymbol{\alpha ~ ( - )}$ \\
\hline EF10 & 26.04 & 25.24 & .66 & .84 \\
\hline EF27 & 25.83 & 25.31 & .69 & .84 \\
\hline EF15 & 26.12 & 25.33 & .61 & .85 \\
\hline EF17 & 25.82 & 24.95 & .65 & .84 \\
\hline EF39 & 26.07 & 25.05 & .64 & .85 \\
\hline EF35 & 25.76 & 25.33 & .63 & .85 \\
\hline EF19 & 26.00 & 25.61 & .52 & .86 \\
\hline EF30 & 25.74 & 25.44 & .56 & .85 \\
\hline
\end{tabular}

Nota $=$ Se han agregado las siguientes abreviaciones de aquí en adelante. Media de escala si el elemento se ha suprimido: Me (-); Varianza de escala si el elemento se ha suprimido: R (-); Correlación total de elementos corregida: C.T; Alfa de Cronbach si el elemento se ha suprimido: $\alpha(-)$ 
La segunda escala se denominó Sistema de supervisión y monitorización de la conducta para el aprendizaje. La escala contiene 7 elementos en los cuales se ha obtenido un Alfa de Cronbach de .86 (ver tabla 3).

Tabla 3. Escala 2 Sistema de supervisión y monitorización de la conducta para el aprendizaje.

\begin{tabular}{|ccccc|} 
& Me (-) & $\mathbf{R}(\boldsymbol{-})$ & C. $\mathbf{T}$ & $\boldsymbol{\alpha}(\boldsymbol{-})$ \\
\hline EF9 & 25.53 & 15.02 & .71 & .83 \\
\hline EF8 & 25.60 & 15.10 & .63 & .84 \\
\hline EF2 & 25.48 & 15.03 & .59 & .85 \\
\hline EF29 & 25.53 & 15.33 & .67 & .84 \\
\hline EF18 & 25.33 & 15.14 & .61 & .84 \\
\hline EF38 & 25.72 & 14.80 & .57 & .85 \\
\hline EF25 & 25.46 & 14.79 & .66 & .84 \\
\hline
\end{tabular}

La tercera escala se denominó Sistema regulador consciente y voluntario de las emociones. La escala contiene 7 elementos en los cuales se ha obtenido un Alfa de Cronbach de .81 (ver tabla 4).

Tabla 4. Escala 3 Sistema regulador consciente y voluntario de las emociones.

\begin{tabular}{|lcccc|}
\hline & Me (-) & R (-) & C.T & $\boldsymbol{\alpha ~ ( - )}$ \\
\hline EF34 & 21.71 & 17.03 & .65 & .77 \\
\hline EF14 & 22.00 & 17.62 & .50 & .80 \\
\hline EF28 & 21.50 & 18.54 & .50 & .80 \\
\hline EF23 & 21.57 & 19.65 & .38 & .82 \\
\hline EF4 & 21.77 & 18.34 & .58 & .79 \\
\hline EF40 & 21.79 & 17.38 & .58 & .78 \\
\hline EF42 & 21.72 & 16.99 & .69 & .77 \\
\hline
\end{tabular}

La cuarta escala se denominó Sistema de verificación de la conducta para el aprendizaje. La escala contiene 4 elementos los cuales han obtenido un Alfa de Cronbach de .68 (ver tabla 5).

Tabla 5. Escala 4 Sistema de verificación de la conducta para el aprendizaje.

\begin{tabular}{|ccccc|}
\hline & Me (-) & R (-) & C.T & $\boldsymbol{\alpha}(\boldsymbol{-})$ \\
\hline EF31 & 12.22 & 5.27 & .56 & .71 \\
\hline EF24 & 12.20 & 5.20 & .63 & .67 \\
\hline EF41 & 12.28 & 5.72 & .55 & .71 \\
\hline EF33 & 12.02 & 5.79 & .52 & .73 \\
\hline
\end{tabular}

La quinta escala se denominó Sistema de regulación consciente de la conducta. La escala contiene 4 elementos los cuales han obtenido un Alfa de Cronbach de .73 (ver tabla 6)

Tabla 6. Escala 5. Sistema de regulación consciente de la conducta.

\begin{tabular}{|ccccc|}
\hline & Me (-) & $\boldsymbol{R}(-)$ & C. $\mathbf{T}$ & $\boldsymbol{\alpha}(-)$ \\
\hline EF22 & 12.38 & 4.71 & .48 & .70 \\
\hline EF20 & 12.07 & 4.65 & .52 & .68 \\
\hline EF21 & 12.47 & 4.58 & .53 & .67 \\
\hline EF12 & 12.27 & 4.74 & .57 & .65 \\
\hline
\end{tabular}

La sexta escala se denominó Sistema de organización de elementos para resolver tareas. La escala contiene 4 elementos los cuales han obtenido un Alfa de Cronbach de .77 (ver tabla 7)

Tabla 7. Escala 6 Sistema de organización de elementos para resolver tareas.

\begin{tabular}{|lcccc|}
\hline & Me (-) & $\mathbf{R}(-)$ & C. $\mathbf{T}$ & $\boldsymbol{\alpha}(-)$ \\
\hline EF6 & 12.12 & 5.83 & .64 & .68 \\
\hline EF7 & 11.91 & 6.36 & .54 & .73 \\
\hline EF1 & 11.76 & 5.99 & .57 & .71 \\
\hline EF37 & 11.86 & 6.09 & .54 & .73 \\
\hline
\end{tabular}

La séptima escala se denominó Sistema ejecutivo para toma de decisiones. La escala contiene 4 elementos los cuales han obtenido un Alfa de Cronbach de .74 (ver tabla 8).

Tabla 8. Escala 7. Sistema ejecutivo para toma de decisiones..

\begin{tabular}{|lcccc|} 
& Me (-) & R (-) & C.T & $\boldsymbol{\alpha}(-)$ \\
\hline EF26 & 11.93 & 4.36 & .60 & .64 \\
\hline EF5 & 11.66 & 4.48 & .54 & .67 \\
\hline EF13 & 11.82 & 4.42 & .56 & .66 \\
\hline EF16 & 11.91 & 4.79 & .43 & .73 \\
\hline
\end{tabular}

La octava escala se denominó Sistema de control consciente de impulsos automáticos. La escala contiene 3 elementos los cuales han obtenido un Alfa de Cronbach de .49 (ver tabla 9).

Tabla 9. Escala 8. Sistema de control consciente de impulsos automáticos.

\begin{tabular}{|lcccc|}
\hline & Me (-) & $\mathbf{R}(-)$ & C. $\mathbf{T}$ & $\boldsymbol{\alpha}(-)$ \\
\hline EF11 & 7.70 & 2.25 & .33 & .36 \\
\hline EF3 & 7.34 & 2.68 & .38 & .29 \\
\hline EF32 & 7.25 & 2.84 & .23 & .51 \\
\hline
\end{tabular}

La Correlación total de elementos corregida, es el coeficiente de homogeneidad corregido, en donde si es cero o negativo se elimina o replantea la pregunta, en el caso de la presente escala, no sería necesario. El Alfa de Cronbach si se elimina el elemento, equivale al valor de Alfa si eliminamos cada uno de los ítems, en el caso de la presente escala, la eliminación del ítem 32, mejoraría el alfa de .49 a .51 , cabe destacar que, a pesar de esta eliminación, la presente escala no podría contar con un correcto nivel de fiabilidad y/o relación entre las preguntas.

\section{Análisis de correlación entre escalas}

En la tabla 10 se pueden observar los resultados del análisis de correlación entre los sistemas propuestos. Se evidencia que existe una correlación positiva en todas las funciones ejecutivas, debido a que los valores corresponden a ser mayores que 0 y no existen variables correlacionadas en sentido inverso, es decir, menores a 0 . 
Tabla 10. Análisis de correlación.

\begin{tabular}{|c|c|c|c|c|c|c|c|c|}
\hline & V1 & V2 & V3 & V4 & V5 & V6 & V7 & V8 \\
\hline Sistema ejecutivo de control atencional para tareas & 1 & & & & & & & \\
\hline \multirow[t]{2}{*}{ Sistema de supervisión y monitorización de la conducta para el aprendizaje } & $.67^{* *}$ & 1 & & & & & & \\
\hline & .00 & & & & & & & \\
\hline \multirow[t]{2}{*}{ Sistema regulador consciente y voluntario de las emociones } & $.52^{* *}$ & $.39^{* *}$ & 1 & & & & & \\
\hline & .00 & .00 & & & & & & \\
\hline \multirow[t]{2}{*}{ Sistema de verificación de la conducta para el aprendizaje } & $.59^{* *}$ & $.66^{* *}$ & $.41^{* *}$ & 1 & & & & \\
\hline & 00 & .00 & .00 & & & & & \\
\hline \multirow[t]{2}{*}{ Sistema de regulación consciente de la conducta } & $.60^{* *}$ & $.64^{* *}$ & $.50^{\star \star}$ & $.59^{* *}$ & 1 & & & \\
\hline & .00 & .00 & .00 & .00 & & & & \\
\hline \multirow[t]{2}{*}{ Sistema de organización de elementos para resolver tareas } & $.50^{\star \star}$ & $.52^{\star *}$ & $.41^{* *}$ & $.47^{* *}$ & $.46^{\star \star}$ & 1 & & \\
\hline & .00 & .00 & .00 & .00 & .00 & & & \\
\hline \multirow[t]{2}{*}{ Sistema ejecutivo para toma de decisiones } & $.56^{* *}$ & $.61^{* *}$ & $.50^{* *}$ & $.49^{* *}$ & $.56^{\star \star}$ & $.44^{\star *}$ & 1 & \\
\hline & .00 & .00 & .00 & .00 & .00 & .00 & & \\
\hline \multirow[t]{2}{*}{ Sistema de control consciente de impulsos automáticos } & $.60^{* *}$ & $.56^{* *}$ & $.59^{\star *}$ & $.54^{\star *}$ & $.58^{\star *}$ & $.49^{* *}$ & $.50^{* *}$ & 1 \\
\hline & 00 & .00 & .00 & .00 & .00 & .00 & .00 & \\
\hline
\end{tabular}

**. La correlación es significativa en el nivel 0,01 (bilateral)

Nota: Se han agregado las siguientes abreviaciones: Sistema ejecutivo de control atencional para tareas (V1); Sistema de supervisión y monitorización de la conducta para el aprendizaje (V2); Sistema regulador consciente y voluntario de las emociones (V3); Sistema de verificación de la conducta para el aprendizaje (V4); Sistema de regulación consciente de la conducta (V5); Sistema de organización de elementos para resolver tareas (V6); Sistema ejecutivo para toma de decisiones (V7); Sistema de control consciente de impulsos automáticos (V8)

\section{Análisis factorial exploratorio}

A continuación, se hizo un análisis factorial exploratorio en donde se encontró que los ítems se organizan en 8 factores con las siguientes cargas factoriales: enlistar los factores (ver tabla 11).

\section{Discusión}

En este artículo se propone una escala en formato de auto reporte para la evaluación de las funciones ejecutivas en estudiantes universitarios. En dicha escala, se propusieron 41 ítems que se subdividen en 8 cargas factoriales y 8 sistemas que engloban las $\mathrm{FE}$ a evaluar. Se ha buscado, que tanto los sistemas propuestos como los ítems, se encuentren enfocados al ámbito universitario, permitiendo así obtener resultados precisos del funcionamiento cerebral frontal en actividades cotidianas que un estudiante del nivel superior puede presentar.

La organización factorial nos invita a pensar que las funciones ejecutivas son sistemas funcionales cerebrales que interactúan entre sí para que el ser humano logre regular su comportamiento y no como elementos aislados anatómicamente. ${ }^{17}$ Esto permite ver a los lóbulos frontales, en específico a la corteza prefrontal como un sistema complejo que abarca a las funciones ejecutivas y las mismas cumplen y participan con varias funciones de control, regulación y planeación eficiente de la conducta humana y ninguna de estas funciones podrían ser entendidas como elementos aislados, sino como sistemas que trabajan y se relacionan con uno o más componentes. ${ }^{18,19,20}$

En cuanto al análisis de consistencia interna, se pudo evidenciar que 7 de los 8 sistemas propuestos, cuentan con un nivel de fiabilidad aceptable. La octava escala, denominada: Sistema de control de impulsos automáticos, contó con un nivel bajo correlación entre varios
Tabla 11. Análisis factorial exploratorio.

\begin{tabular}{|c|c|c|c|c|c|c|c|c|}
\hline & \multicolumn{8}{|c|}{$\begin{array}{l}\text { Matriz de componente rotado } \\
\text { Componente }\end{array}$} \\
\hline & 1 & 2 & 3 & 4 & 5 & 6 & 7 & 8 \\
\hline EF10 & .69 & & & & & & & \\
\hline EF27 & .66 & & & & & & & \\
\hline EF15 & .65 & & & & & & & \\
\hline EF17 & .63 & & & & & & & \\
\hline EF39 & .61 & & & & & & & \\
\hline EF35 & .54 & & & & & & & \\
\hline EF19 & .52 & & & & & & & \\
\hline EF30 & .49 & & & & & & & \\
\hline EF9 & & .77 & & & & & & \\
\hline EF8 & & .69 & & & & & & \\
\hline EF2 & & .61 & & & & & & \\
\hline EF29 & & .58 & & & & & & \\
\hline EF18 & & .50 & & & & & & \\
\hline EF38 & & .49 & & & & & & \\
\hline EF25 & & .48 & & & & & & \\
\hline EF42 & & & .74 & & & & & \\
\hline EF34 & & & .73 & & & & & \\
\hline EF14 & & & .67 & & & & & \\
\hline EF40 & & & .65 & & & & & \\
\hline EF4 & & & .64 & & & & & \\
\hline EF28 & & & .47 & & & & & \\
\hline EF23 & & & .32 & & & & & \\
\hline EF31 & & & & .69 & & & & \\
\hline EF24 & & & & .65 & & & & \\
\hline EF41 & & & & .58 & & & & \\
\hline EF33 & & & & .47 & & & & \\
\hline EF22 & & & & & .6 & & & \\
\hline EF20 & & & & & .5 & & & \\
\hline EF21 & & & & & .5 & & & \\
\hline EF12 & & & & & $.4 \varepsilon$ & & & \\
\hline EF6 & & & & & & .78 & & \\
\hline EF7 & & & & & & .68 & & \\
\hline EF1 & & & & & & .65 & & \\
\hline EF37 & & & & & & .65 & & \\
\hline EF26 & & & & & & & .67 & \\
\hline EF5 & & & & & & & .67 & \\
\hline EF13 & & & & & & & .53 & \\
\hline EF16 & & & & & & & .50 & \\
\hline EF11 & & & & & & & & .55 \\
\hline EF3 & & & & & & & & .47 \\
\hline EF32 & & & & & & & & -.38 \\
\hline
\end{tabular}


elementos, es por esto que se eliminarían los ítems 3, 11 y 32 para la escala propuesta. Del mismo modo, dentro de la cuarta escala denominada: Sistema de verificación de la conducta para el aprendizaje, se obtuvo un Alfa de Cronbach de .68, empero, con la eliminación del ítem 33 la escala de fiabilidad subió a .73. En cuanto a los demás sistemas la eliminación de cualquier ítem no modificaría o aumentaría el estadístico de fiabilidad lo cual nos permite asegurar que se obtuvieron buenos resultados.

Se encontró una correlación estadísticamente significativa entre las escalas con una direccionalidad positiva, en donde supone una determinación absoluta entre variables, debido a que los valores corresponden a ser mayores que 0 y no existen variables correlacionadas en sentido inverso, es decir, menores a 0 . Lo cual nos invita a reflexionar que las funciones ejecutivas propuestas en esta escala tienen una misma direccionalidad en su interpretación.

Dentro del análisis factorial exploratorio, la matriz de componentes nos informa de la relación entre las variables y mediante su rotación nos permite identificar cada una de las dimensiones latentes extraídas, esto nos lleva a evidenciar que las mismas han convergido en 8 grupos que hemos denominado como escalas. Dentro de las 8 escalas se encuentran ítems que evalúan varias funciones ejecutivas, es por esto por lo que se nombró a cada escala con un respectivo sistema que abarque las funciones ejecutivas a evaluar. Por ejemplo: El Sistema ejecutivo de control atencional para tareas fue denominado así, debido a que los ítems seleccionados en esta escala evalúan: la memoria de trabajo, la planificación e iniciativa y sus ítems tienen en común la concentración y atención en actividades universitarias.

El Sistema de supervisión y monitorización de la conducta para el aprendizaje, ha sido denominado así debido a que evalúa la monitorización, planificación e iniciativa y sus ítems tienen en común el realizar o terminar actividades universitarias de manera independiente. El Sistema regulador consciente y voluntario de las emociones, fue denominado de esa manera ya que evalúa el control emocional e inhibición y sus ítems tienen en común el control emocional. El Sistema de verificación de la conducta para el aprendizaje, fue denominado de esta manera ya que evalúa la planificación y la memoria de trabajo y sus ítems tienen en común la verificación del cumplimiento de objetivos. El Sistema de regulación consciente de la conducta ha sido denominado así ya que evalúa la inhibición, monitorización y memoria de trabajo, sus ítems tienen en común el estado de actividad basado en la intención del individuo.

El Sistema de organización de elementos para resolver tareas ha sido nombrado así, ya que evalúa la organización de materiales, sus ítems tienen en común el control consciente de la organización. El Sistema ejecutivo para la toma de decisiones, fue denominado así ya que evalúa la flexibilidad cognitiva e iniciativa, sus ítems tienen en común la toma de decisiones y solución de problemas. El Sistema de control consciente de impulsos automáticos, se ha nombrado así ya que evalúa la inhibición y flexibilidad cognitiva, sus ítems tienen en común el control consciente del comportamiento y adaptación.

La propuesta realizada vendría a ser un interesante aporte dentro de la investigación sobre funciones ejecutivas ya que abre paso a la evaluación en un contexto poco explorado que podrá visibilizar la problemática de varios estudiantes y sus dificultades para llevar a cabo sus actividades, tareas, planes $\mathrm{u}$ objetivos universitarios. Además, pone en relieve el control emocional que un estudiante pueda tener ante la presión académica o el manejo de tareas con actividades del hogar, entre otras. Por otro lado, se puede llegar a evidenciar dificultades o inmadurez en las funciones ejecutivas derivando en problemas de índole comportamental o cognitivo.

Además, es importante recalcar que los resultados de esta investigación puedan generar nuevas hipótesis a estudiarse, por ejemplo, la utilización de la presente escala para estudios comparativos descriptivos o correlacionales que evalúen variables como el estrés académico, rendimiento académico, planificación de tareas, cumplimiento de objetivos, entre otros. Además, se pone a consideración la utilización de la presente escala para evaluar a estudiantes universitarios en niveles que se han considerado como frecuentes en la deserción académica, logrando determinar si los alumnos cuentan con las estrategias y/o habilidades cognitivas necesarias; lo cual daría paso a la implementación de mejores herramientas para evitar la deserción y/o el mal desempeño académico. Se logra observar la cantidad de nuevas y múltiples investigaciones que podrían partir de la utilización de la escala propuesta.

Como limitaciones de la presente investigación se deben mencionar las siguientes: en primer lugar, se debe de considerar el carácter subjetivo en la aplicación de la prueba de auto reporte ya que puede estar sesgado por la percepción del individuo sobre su realidad conductual; en segundo lugar, la muestra pertenece a una ciudad determinada de Latinoamérica. No obstante, esta situación nos motiva a continuar en nuevos procesos de investigación en los cuales podamos identificar el comportamiento psicométrico de esta escala para valorar funciones ejecutivas.

\section{Referencias}

1. Tirapu-Ustárroz, J., García-Molina, A., Luna-Lario, P., Roig-Rovira, T., \& Pelegrín-Valero, C. Modelos de funciones y control ejecutivo (II). Revista de Neurología. 2008; 46 (12): 742-750.

2. González, M. Desarrollo neuropsicológico de las funciones ejecutivas en la edad preescolar. México: Editorial Manual Moderno; 2015. 
3. Mujica, A. El Llamado Síndrome del Lóbulo Frontal, actualmente llamado Síndrome Disejecutivo. Revista Argentina de Clínica Neuropsiquiátrica. 2011; 17(1): 42-47.

4. Portellano, J., \& García, A. Neuropsicología de la atención, las funciones ejecutivas y la memoria. España: Editorial SÍNTESIS, S.A.; 2014.

5. Bechara, A., Damasio, A., Damasio, H., \& y Anderson, S. Insensitivity to future consequences following damage to human prefrontal cortex. Cognition. 1994; 50 (1-3): 7-15.

6. Gardner, H. Intelligence Reframed: Múltiple Intelligences for the 21st Century. Barcelona: Paidós; 1999.

7. Goldberg, E. El cerebro ejecutivo. Lóbulos frontales y mente civilizada. Barcelona: Editorial Crítica; 2002.

8. Miyake, A., Friedman, N., Emerson, M., Howerter, W., \& Wager, T. The unity and diversity of executive functions and their contributions to complex "frontal lobe". Cognitive Psychology. 2000; 41 (1): 49-100.

9. Shimamura, A. The role of the prefrontal cortex in dynamic filtering. Psychobiology. 2000; 28: 28-207.

10. Ardila, A., \& Rosselli, M. Neuropsicología Clínica. México: Editorial Manual Moderno; 2007.

11. Ramos-Galarza, C., Acosta-Rodas, P., BolañosPasquel, M., \& Lepe, N. The role of executive functions in academic performance and behaviour of university students. Journal of Applied Research in Higher Education. 2019; 1-12.

12. Villegas, C. Relación entre funciones ejecutivas y estrés académico en estudiantes universitarios (Tesis de grado de Psicología). Pontificia Universidad Católica del Ecuador, Quito-Ecuador; 2019.
13. Nunes, A., Ramos, M., \& da Silva, C. Instrumentos para la evaluación de funciones ejecutivas: revisión sistemática de los cinco años anteriores. Avaliação Psicológica. 2019; 18(1), 96-107.

14. García, A. Evaluación de las funciones ejecutivas. Madrid: Editorial Síntesis; 2018.

15. Bojórquez, J., López, L., Hernández, M., \& Jiménez, E. Utilización del alfa de Cronbach para validar la confiabilidad de un instrumento de medición de satisfacción del estudiante en el uso del software Minitab. 11th Latin American and Caribbean Conference for Engineering and Technology; 2013; Cancún. p. 1-9.

16. Celina, H., \& Campo, A. Aproximación al uso del coeficiente alfa de Cronbach. Revista Colombiana de psiquiatría. 2005 ; 34(4): 572-580.

17. Flores, J., \& Ostrosky-Solís, F. Neuropsicología de Lóbulos Frontales, Funciones Ejecutivas y Conducta Humana. Revista Neuropsicología, Neuropsiquiatría y Neurociencias. 2008; 8(1): 47-58.

18. Flores, J., \& Ostrosky-Solís, F. Neuropsicología de Lóbulos Frontales, Funciones Ejecutivas y Conducta Humana. Revista Neuropsicología, Neuropsiquiatría y Neurociencias. 2008; 8(1): 47-58.

19. Rosselli, M., Jurado, M., \& Matute, E. Las Funciones Ejecutivas a través de la Vida. Revista Neuropsicología, Neuropsiquiatría y Neurociencias. 2008; 8(1): 23-46.

20. Tirapu-Ustárroz, J., García-Molina, A., Ríos-Lago, M., \& Ardila, A. Neuropsicología de la corteza prefrontal y las funciones ejecutivas. Madrid: Editorial Viguera; 2012. 
Anexo 1.

Escala propuesta para evaluar $\mathrm{FE}$

Sistema de control atencional, conducta organizada e

intencionada

10. Tengo buena concentración.

27. Me es fácil concentrarme en mis actividades universitarias.

15. Me concentro en mis actividades universitarias, dejando de lado las cosas irrelevantes.

17. Soy capaz de mantener la atención en una actividad.

39. Mantengo buenos hábitos de estudio.

35. Me resulta fácil estar dispuesto/a iniciar las tareas de la universidad sólo con proponérmelas.

19. Planifico mis tareas universitarias con tiempo.

30. Puedo regresar con facilidad a una tarea de la universidad después de tomar un descanso.

Sistema de supervisión y

9. Logro realizar exitosamente mis trabajos de la universidad.

monitorización de la conducta

para el aprendizaje

8. Puedo realizar las tareas universitarias de forma independiente y sin ayuda de los demás.

2. Puedo terminar una tarea universitaria cuando es muy larga.

18. Puedo realizar mis trabajos sin que alguien me supervise.

29. Puedo realizar apropiadamente actividades o tareas universitarias que tengan más de un paso.

25. Soy capaz de comenzar con mis responsabilidades universitarias, sin necesidad de que alguien me indique que debo hacerlo.

38. Termino mis tareas universitarias a tiempo.

Sistema regulador consciente 34. Mantengo la calma con facilidad.

y voluntario de las emociones

14. Cuando me enfado puedo calmarme con facilidad.

28. Suelo ser prudente con las cosas que digo a otras personas.

23. Puedo anticipar las consecuencias de mis actos.

4. Controlo adecuadamente mis emociones en alguna situación universitaria.

40. Tengo un estado de ánimo estable.

42. Soy capaz de controlar mis emociones.

Sistema de verificación de la

31. Reviso la ortografía y redacción de mis tareas universitarias antes de finalizarlas.

conducta para el aprendizaje

24. Verifico que mis tareas universitarias estén bien realizadas y sin errores, antes de presentarlas al profesor.

41. Al finalizar una actividad universitaria, verifico que haya logrado lo planificado.

33. Recuerdo llevar a casa las tareas, materiales o trabajos de la universidad.

Sistema de regulación

22. Dejo hablar a mis compañeros de aula, sin hacer interrupciones.

consciente de la conducta

20. Me es fácil comportarme adecuadamente en las reuniones sociales.

21. Cuando mis profesores me lo piden, puedo dejar con facilidad de hacer algo que me distrae.

12. Sigo de manera adecuada las instrucciones que se me dan en la universidad.

Sistema de organización de 6. Tengo mis cosas en el lugar adecuado y en orden.

elementos para resolver tareas

7. Tengo facilidad para encontrar rápidamente mis materiales al buscarlos en mi cuarto o escritorio.

1. Tengo facilidad para recoger y dejar ordenadas mis cosas cuando se me pide que lo haga.

37. Recojo mi desorden sin que otros compañeros de la universidad lo hagan por mí.

Sistema ejecutivo para toma 26. Puedo tomar decisiones sin dificultad, incluso ante las cosas más complicadas.

de decisiones

5. Tengo la capacidad para tomar decisiones en forma independiente en los diferentes retos que se presenta en la universidad.

13. Tengo la capacidad para resolver problemas en la universidad como en mi vida personal.

16. Me resulta fácil cambiar de una tarea a otra.

Sistema de control consciente

11. Puedo estar quieto/a y tranquilo/a mientras espero mi turno en alguna situación de la universidad.

de impulsos automáticos

3. Actúo siempre pensando y reflexionando las consecuencias de mis actos.

32. Puedo adaptarme con facilidad a los cambios en mis rutinas, a nuevos profesores, nuevos horario, a cambios en los planes familiares o de la universidad. 